\title{
BMJ Open Testing the effectiveness of a weight loss intervention to enhance self- regulation in adults who are obese: protocol for a randomised controlled trial
}

Kerstin Frie (1) , Jamie Hartmann-Boyce (D) , Susan A Jebb (1) , Paul Aveyard (1)

To cite: Frie K, HartmannBoyce J, Jebb SA, et al. Testing the effectiveness of a weight loss intervention to enhance self-regulation in adults who are obese: protocol for a randomised controlled trial. BMJ Open 2019;9:e031572. doi:10.1136/ bmjopen-2019-031572

- Prepublication history and additional material for this paper are available online. To view these files, please visit the journal online (http://dx.doi. org/10.1136/bmjopen-2019031572).

Received 10 May 2019 Revised 26 0ctober 2019 Accepted 11 November 2019

D Check for updates

(c) Author(s) (or their employer(s)) 2019. Re-use permitted under CC BY. Published by BMJ.

Nuffield Department of Primary Care Health Sciences, University of Oxford, Oxford, UK

Correspondence to

Kerstin Frie;

kerstin.frie@phc.ox.ac.uk

\section{ABSTRACT}

Introduction Previous trials finding an effect of self-monitoring on weight loss have considered the effect to be mediated by self-regulatory processes. However, a qualitative think-aloud study asking people to record thoughts and feelings during weighing showed that self-regulation occurs only rarely without further instruction. The aim of this trial is to test a novel intervention guiding people through the self-regulatory processes to see whether it facilitates weight loss.

Methods and analyses A parallel group, randomised controlled trial will be conducted to test the concept that a self-regulation intervention for weight loss increases weight loss compared with daily self-weighing without further support. One hundred participants with a body mass index $\geq 30 \mathrm{~kg} / \mathrm{m}^{2}$ will be randomised to either the control or intervention group. The control group will be asked to weigh themselves daily for 8 weeks, the intervention group will be encouraged to follow the self-regulation intervention. They will be prompted to weigh daily, track their weight using an app, plan daily actions for weight loss and reflect on their action plans on a weekly basis. This self-regulation cycle will allow them to experiment with different weight loss strategies and identify effective and sustainable actions. Primary and process outcomes will be measured at baseline and 8 weeks' follow-up. Linear regression analysis of the primary outcome, weight change, will assess the early effectiveness of the intervention. The process outcomes liking, perceived effectiveness, as well as usage and barriers with regard to the self-regulation intervention, will be assessed through qualitative analysis of follow-up interviews and quantitative analysis of adherence rates and responses to a final questionnaire.

Ethics and dissemination This trial was reviewed and approved by the NHS National Research Ethics Committee and the Health Research Authority (reference number: 18/SC/0482). The findings of the trial will be published in peer reviewed journals and presented at conferences.

Trial registration number ISRCTN14148239, prerecruitment.

Protocol version Version 1.1, 7 December 2018
Strengths and limitations of this study

- This trial will provide preliminary evidence for whether a remotely delivered and iterative selfregulation intervention can enhance weight loss.

- The intervention addresses specific barriers to selfregulation, identified in a previous qualitative study of experiences of self-weighing.

- Process evaluation measures will assess participants liking, usage and perceived effectiveness of the intervention to enable future improvements.

- Blinding might be compromised as participants might be aware that they were assigned to the control or treatment group.

- The study is designed as a 'proof-of-concept' trial and longer-term studies will need to evaluate the effectiveness of the intervention as a weight management tool.

\section{INTRODUCTION}

Self-monitoring of weight is often employed in multicomponent weight loss interventions, as evidence suggests it aids weight loss. ${ }^{1-5}$ The weight loss effect has often been ascribed to a self-regulation mechanism, based on the hypothesis that self-monitoring triggers selfregulation. ${ }^{14-6}$ In this context, self-regulation occurs in iterative cycles, starting by (1) contextualising the weight with previous measurements and goals, thus providing (2) an opportunity to reflect on previous behaviour and reinforce successful actions, enabling (3) the planning of actions to reach the goal, followed by (4) the performance of planned actions. ${ }^{6-8}$ This cycle of processes allows for experimentation with different weight loss techniques, helping the user to build a personal portfolio of effective and sustainable strategies. ${ }^{910}$

A study employing self-weighing as a standalone intervention did not find a significant 
weight loss effect, raising the question whether selfregulation is performed naturally after weighing or whether additional weight loss treatment components are necessary. ${ }^{11}$ We addressed this question in a thinkaloud study, where twenty-four participants were asked to record their thoughts and feelings during daily weighing for 8 weeks, without being prompted to self-regulate. ${ }^{12}$ On $90 \%$ of occasions, participants contextualised their weight measurement, and on $58 \%$ participants reflected on previous behaviours. Only on $20 \%$ of occasions did participants plan actions and specific action planning, defining a concrete action and time plan, was rare $(6 \%)$. The frequency of specific action planning was, however, significantly predictive of weight loss. Hence, the study provided support to the notion that completing the last step of the self-regulation process can elicit weight loss. However, the think-aloud study also showed that selfregulation does not occur autonomously, and that people need support in developing self-regulation skills, especially action planning. Self-regulation, once learnt, has the potential to be a weight loss strategy that is performed autonomously and sustainably. It provides an opportunity for remote weight loss interventions that do not require resource-intensive support from healthcare providers.

Several weight loss interventions including selfregulation components have been developed over the years. Some educate their users about self-regulation theory without providing active support for the key component—specific action planning. ${ }^{1} 41314$ Others support users in action planning, but fail to imitate the iterative nature of self-regulation, as they do not allow the usage of self-monitoring feedback for adaptations to the action plans,${ }^{15-18}$ thus disabling self-regulation. Some interventions guide participants through iterative reformulation of action plans following self-monitoring feedback, but are delivered through face-to-face sessions, ${ }^{19} 20$ which are resource-intensive and not easily rolled out at large scale. Other interventions incorporating action planning dictate which actions participants are supposed to follow, ${ }^{15} 2122$ which might reduce goal ownership, a significant predictor of goal engagement and attrition. ${ }^{23}{ }^{24}$ Notwithstanding these critiques, seven of the eleven studies cited here found significant weight loss, suggesting that components targeting the self-regulation cycle can enhance weight loss, encouraging further work in this area. Our critiques highlight the need for an intervention that guides participants through the whole and iterative self-regulation process in an autonomous, low-cost and scalable manner. Furthermore, since many interventions add self-regulation elements to a broader spectrum of weight loss treatment components, ${ }^{14} 1725-27$ a study testing self-regulation as a standalone intervention is needed to investigate whether iterative self-regulation is sufficient to achieve weight loss.

With this study we aim to test the proof of concept of an intervention aiming to address this gap in the literature. The PREVAIL intervention (People REgulating themsel Ves to Achieve weIght Loss) is a weight loss programme guiding people through the iterative self-regulation process. It encourages users to experiment with different weight loss approaches, and use the self-regulation mechanism to find their ideal set of tools.

\section{Objectives}

The primary objective of this trial is to test the concept whether an intervention which trains individuals in selfregulatory processes, aids early weight loss in comparison to unsupported daily weighing. Other objectives pertain to the evaluation of usage and effectiveness of the self-regulation intervention components, as well as the qualitative analysis of participant experiences of the intervention.

\section{METHODS}

\section{Study design and setting}

An individually randomised, two arm, parallel group design will be employed, assessing superiority of the selfregulation intervention over daily self-weighing alone. Participation will last 8 weeks. This length was deemed sufficient to assess early effectiveness of the intervention, as previous studies have been able to detect weight loss effects after 2 months. ${ }^{28}$ If the results are promising, the data will provide good evidence to justify conducting a longer-term randomised controlled trial. Participants will attend two study visits, one at baseline and one after the end of the eighth week of the intervention. The primary outcome will be weight change. The study will take place in Oxfordshire, UK, and run between April and October 2019.

\section{Recruitment}

Two to four general practitioner (GP) practices around Oxford, UK, will function as participant identification centres and search their health records to identify suitable patients for the trial (age $\geq 18$ years, body mass index $(\mathrm{BMI}) \geq 30 \mathrm{~kg} / \mathrm{m}^{2}$ ). The GP will screen the search list and exclude patients who would be inappropriate to invite, including terminally ill or violent patients. Suitable patients will be sent an invitation letter from their GP. They will be encouraged to contact the research team if they are interested in taking part. GPs may also identify suitable patients during routine consultations. We will ask practices not to refer participants to commercial weight loss programmes, other obesity clinics or bariatric surgery, while they are enrolled as participants in this trial.

\section{Eligibility criteria}

\section{Inclusion criteria}

- Participant is willing and able to give informed consent.

- Aged 18 years or above.

- $\mathrm{BMI} \geq 30 \mathrm{~kg} / \mathrm{m}^{2}$.

- Owns an Apple or Android smartphone.

\section{Exclusion criteria}

The participant may not enter the study if any of the following apply: 
- Unable to understand English.

- Unable to follow all intervention procedures for a period of more than four consecutive days.

- Currently self-monitoring body weight more than once a week.

- Currently or within 3 months of study entry attended a weight management programme or currently participating in another weight loss study.

- Lost more than $5 \%$ of current body weight in the last 6 months.

- Prior bariatric surgery or scheduled for bariatric surgery.

- Pregnant or planning to become pregnant during the course of the study.

- Have an electronic medical implant, such as a pacemaker.

- Have ever had or been diagnosed with an eating disorder.

- People that the GP judges not able to meet the demands of either treatment programme or measurement schedule. This may include severe medical problems not listed above.

\section{Participant flow}

\section{Screening}

People who are interested in taking part will contact the research team. The research team will then discuss study participation by telephone or email and undertake screening. If the person appears eligible and would like to attend a baseline visit, the research team will offer an appointment at a local venue. The participants will be emailed a participant information sheet (PIS).

\section{Baseline}

When the participants attend the baseline appointment, a member of the research team will seek informed consent and check eligibility for inclusion in the study by measuring height and weight for BMI calculation. The participants will be asked to complete an online questionnaire, capturing demographics (ie, age, gender, ethnicity) and previous experiences with self-weighing. Participants will be randomised and receive instructions for the assigned intervention. The researcher will provide participants with a body scale for the duration of the trial. A follow-up appointment will be scheduled for after the completion of the intervention period.

\section{Follow-up}

The aim of the follow-up appointment is to assess the outcomes of the trial. The research team will email participants in advance to remind them of the meeting. In the intervention group, this email will also contain a final questionnaire, asking participants about the usefulness of each of the intervention components and an overall rating of the intervention.

The appointment will be scheduled at a local venue and conducted by a member of the research team.

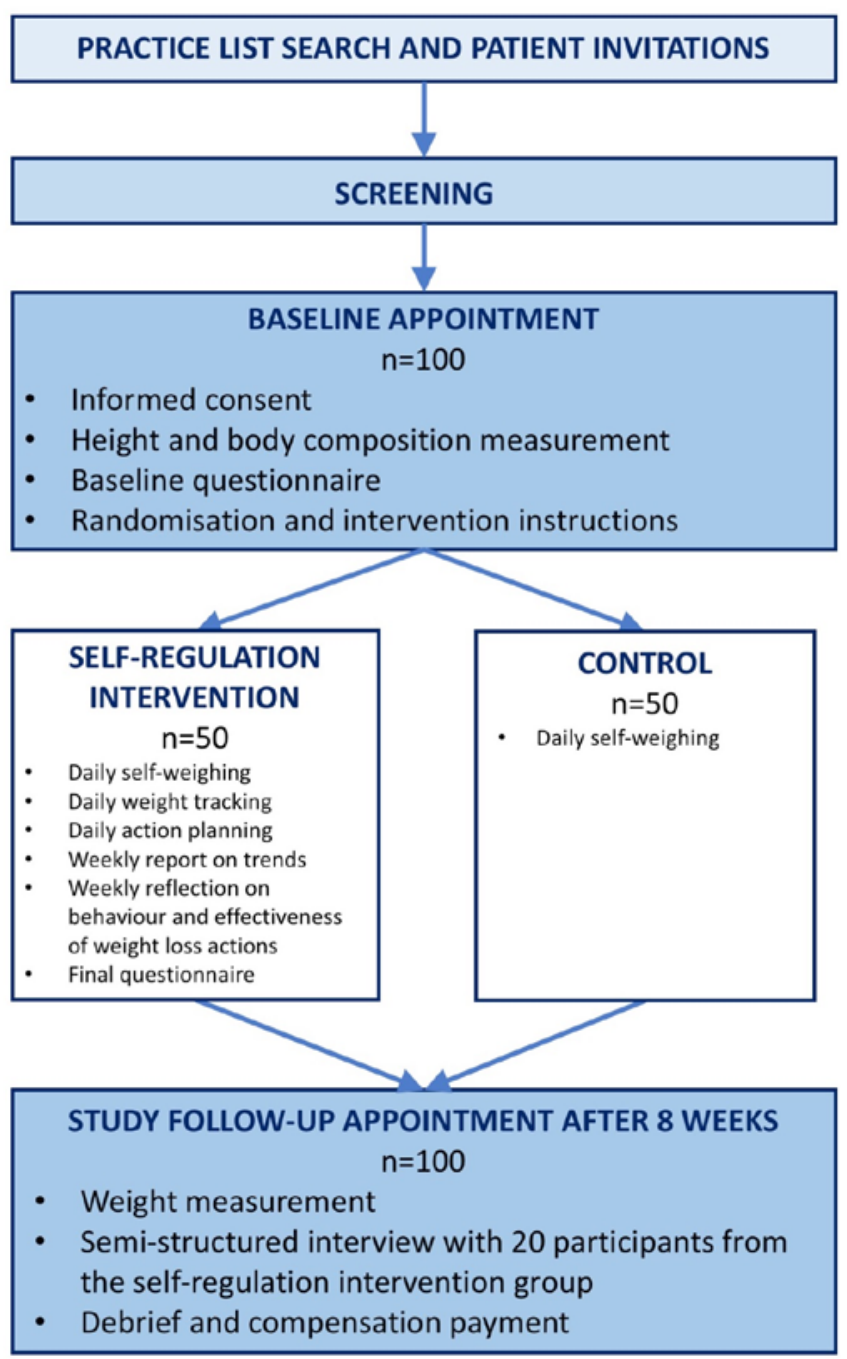

Figure 1 Study flow chart.

Participants will be asked to return the body scales. The researcher will measure participants' weight.

Twenty participants in the intervention group will be invited to participate in a semi-structured interview at the end of the follow-up meeting. The interviewees will be sampled by the lead researcher with the aim to reflect different levels of adherence, weight change and responses to the final questionnaire. Participants will be asked about their experiences with self-weighing, their liking of the intervention components and perceived barriers to engaging with the intervention. Interviews will be recorded, transcribed and analysed. A study flow chart is displayed in figure 1 .

\section{Sample size}

We intend to recruit 100 participants, which is sufficient to detect a $1.5 \mathrm{~kg}$ difference between conditions, at $90 \%$ power and 5\% type I error rate, while allowing for a $20 \%$ drop-out rate. Variance of weight change was based on results of a similar trial, ${ }^{28}$ which reported a SD of $2.13 \mathrm{~kg}$ at 2 months' follow-up. 


\section{Randomisation and blinding}

All eligible participants will be randomised with an allocation ratio of 1:1 to the intervention or control group. A randomisation sequence, stratified by GP and using block randomisation with randomly varying block sizes of 2 and 4 will be generated using a computer algorithm. Allocations will be concealed in numbered, sealed, opaque envelopes by an independent researcher in the department and handed to the researcher who will conduct the baseline visits.

Due to the nature of this trial, it will be difficult to blind participants to the treatment allocation. We will aim to make it as opaque as possible by presenting daily weighing as an intervention to control group participants, as done in previous trials. ${ }^{29}$ The researchers conducting baseline and follow-up will perform data analysis, and will not be blinded to treatment allocation. The primary outcome, weight change, will be measured objectively. Adherence to self-regulation steps will be measured objectively through the frequency of weight logs and completed questionnaires in control and intervention group. The evaluations of treatment components in the final questionnaire will be measured without researchers' input and analysed quantitatively. Blinding of the researcher who conducts and analyses the semi-structured interviews with intervention group participants will not be possible.

\section{Intervention and control}

Intervention

Participants will be asked to weigh themselves every morning after waking using standard body scales (Etekcity, California, USA) provided to them. In addition, they will be prompted to complete tasks which speak to the different steps of the self-regulation process, including (1) contextualising the weight measurement, (2) reflecting on behaviour and (3) planning weight loss actions. The individual tasks and their development are described in more detail below. Input from members of the public was sought at several stages of the intervention development.

1. Contextualising: In the think-aloud study participants struggled memorising daily measurements and keeping an overview of their weight loss progress, which impeded their ability to use weight measurements as constructive feedback. Participants stated they would have benefited from a weight tracking tool. Therefore, to support participants in contextualising their weight measurements, we will encourage them to use the app 'Weight Loss Tracker, BMI' by aktiWir GmbH. Research shows that digital tracking devices can significantly increase adherence to self-monitoring, ${ }^{30} 31$ perhaps because the visualisation of progress and feedback on weight loss success provides motivation and keeps users on track with their goals. ${ }^{32}$

2. Reflecting: The think-aloud study showed that participants struggled to interpret day-to-day weight changes due to daily fluctuations that were not caused by fat loss or gain. ${ }^{12}$ We therefore decided to encourage reflection on a weekly rather than daily basis. Participants will receive weekly emails with feedback on their weekly weight trends, asking them to complete an online questionnaire (Qualtrics, USA). The questionnaire will prompt reflection on the relationship between behaviours performed throughout the week and weight change observed. Participants will be asked to use this insight to evaluate the use of the weight loss actions they had performed throughout the week.

3. Action Planning: We aimed to strike a balance between ensuring that participants choose appropriate actions and allowing them to choose actions themselves, as lack of goal ownership predicts attrition. ${ }^{23}$ Participants will therefore choose one weight loss action per day from a list of 53 actions (see online supplementary appendix 1). To create the actions list, we first identified weight loss actions from effective weight loss interventions in the literature. These were reviewed, adapted and complemented during iterative brainstorming sessions with an interdisciplinary expert team, comprised of dietitians, GPs and psychologists. The rationale for daily action plans was twofold: (1) allowing participants to adapt their actions flexibly to their day and (2) giving participants exposure to a wider range of strategies. Based on action planning and implementation intention research, which shows that the specificity of action plans increases likelihood of implementation, ${ }^{33-35}$ we will ask participants to specify where, when and how they will perform their chosen action, which cues they will use, which barriers they might experience and how they will deal with them.

As participants only reflect on their behaviour on a weekly basis, we grouped the actions conceptually into seven categories, five of which cover diet-related actions and two of which cover physical activity-related actions. Participants will be asked to choose one category at the beginning of the week, and choose daily actions from this category for the rest of the week. Weekly behaviour reflection will therefore focus on the effectiveness of a category of actions. Taken together, the reflection and action planning process shall enable participants to experiment with different weight loss approaches and decide on their effectiveness and usefulness based on trends in weight data. At the beginning of weeks two through eight of the intervention, participants will be prompted to commit to some of the actions of previous weeks, as the performance of several weight loss actions increases chances for weight loss. Action planning support will be provided through daily questionnaires (Qualtrics, USA), which will be sent to participants every morning via email.

At baseline, participants will receive detailed instructions about the intervention with the aid of a manual which they may take home afterwards. The manual will explain the rationale of self-regulation and experimentation to participants and give tips on how to best weigh on a daily basis. It will also provide information on the different weight loss actions participants can try throughout the study. Participants will also be given an 


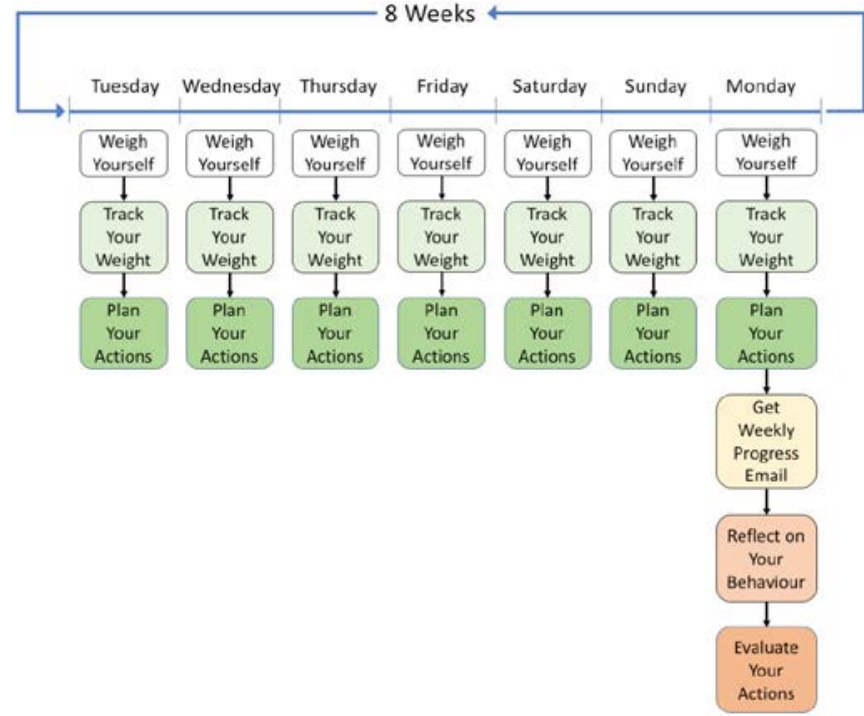

Figure 2 Intervention procedure as depicted in study manual.

action diary, in which they will be asked to record their performed weight loss actions.

We will call participants at the end of the first and fourth week to ask about and solve any technical problems that may have arisen (eg, with the functioning of the scales or the receiving of questionnaires). A figure from the study manual depicting the intervention procedure is displayed in figure 2. The Template for Intervention Description and Replication (TIDieR) checklist ${ }^{36}$ for the PREVAIL intervention and the comparator is reported in table 1 .

\section{Control}

Participants in the control group will be instructed to selfweigh daily and see what daily weighing motivates them to do. They will not receive any further instructions. By using this comparator group, we want to test whether the self-regulation process can enhance self-weighing to be an effective weight loss tool. Participants will receive smart scales (BodyTrace, New York) which are equipped with a SIM card and automatically transfer measurements to a secure server via the $3 \mathrm{G} / 4 \mathrm{G}$ network. This will allow us to assess adherence to daily weighing in the control group.

\section{Patients and public involvement}

Members of the public were involved in the design of the study at several stages. After creating the invitation letter, PIS, informed consent form, semi-structured interview guide and the baseline, daily, weekly and follow-up questionnaires for the PREVAIL intervention, we asked members of the public for feedback. Our department has a panel of $>100$ members of the public with an interest in weight management. Based on phone calls with members of this panel we were able to make the materials clearer and more concise. The manual, explaining the intervention in detail, was further discussed with members of the panel in a focus group session. As a result of this focus group session, the manual was professionally edited to be shorter. We also added figures and graphs to present the procedures of the study more visually. The panel helped to revise the explanations of the different action plans.

A test run of 4 weeks was conducted with five members of the department, who are not otherwise involved in this study. They provided feedback on the running of the intervention which, among other outcomes, led to the creation of a reminder email which will be sent out before the start of the intervention.

No members of the public will be involved in conducting or analysing the study. However, we will gather input on the most suitable and effective ways to disseminate our research findings to the public.

\section{Outcomes}

Primary outcome

- Change in body weight between baseline and follow-up by condition.

\section{Secondary outcomes (process evaluation)}

- Adherence to self-regulation steps, assessed through weight records and daily action planning/weekly evaluation questionnaires.

- Test moderators of effectiveness: adherence measures, highest educational qualification, liking of weighing at baseline, overall rating of intervention in final questionnaire.

- Perceived effectiveness of intervention and liking of intervention features based on final questionnaire and follow-up interviews.

- Barriers and unmet needs for successful weight loss, assessed in follow-up interviews.

\section{Measurements}

A schedule of measurements can be found in table 2 .

\section{Physical measurements}

Participants' height will be measured at baseline to the nearest $0.1 \mathrm{~cm}$ using a stadiometer. Weight will be measured both at baseline and follow-up using a digital scale (SC-240 MA, Tanita Japan). Weight will be recorded to the nearest $0.1 \mathrm{~kg}$.

\section{Process evaluation measures Adherence measures}

For the control group, adherence to daily weighing will be measured by calculating the proportion of days for which we have a recorded weight measurement on the BodyTrace server.

For the intervention group, adherence to daily weighing will be assessed by calculating the proportion of days for which a weight was recorded in the weight tracking app or in the daily action planning questionnaire. Adherence to weight-tracking will be calculated as the proportion of days for which a weight measurement was recorded in the app. Adherence to action planning and reflection will be measured by calculating the proportion of days on which the respective questionnaires were completed. An overall 

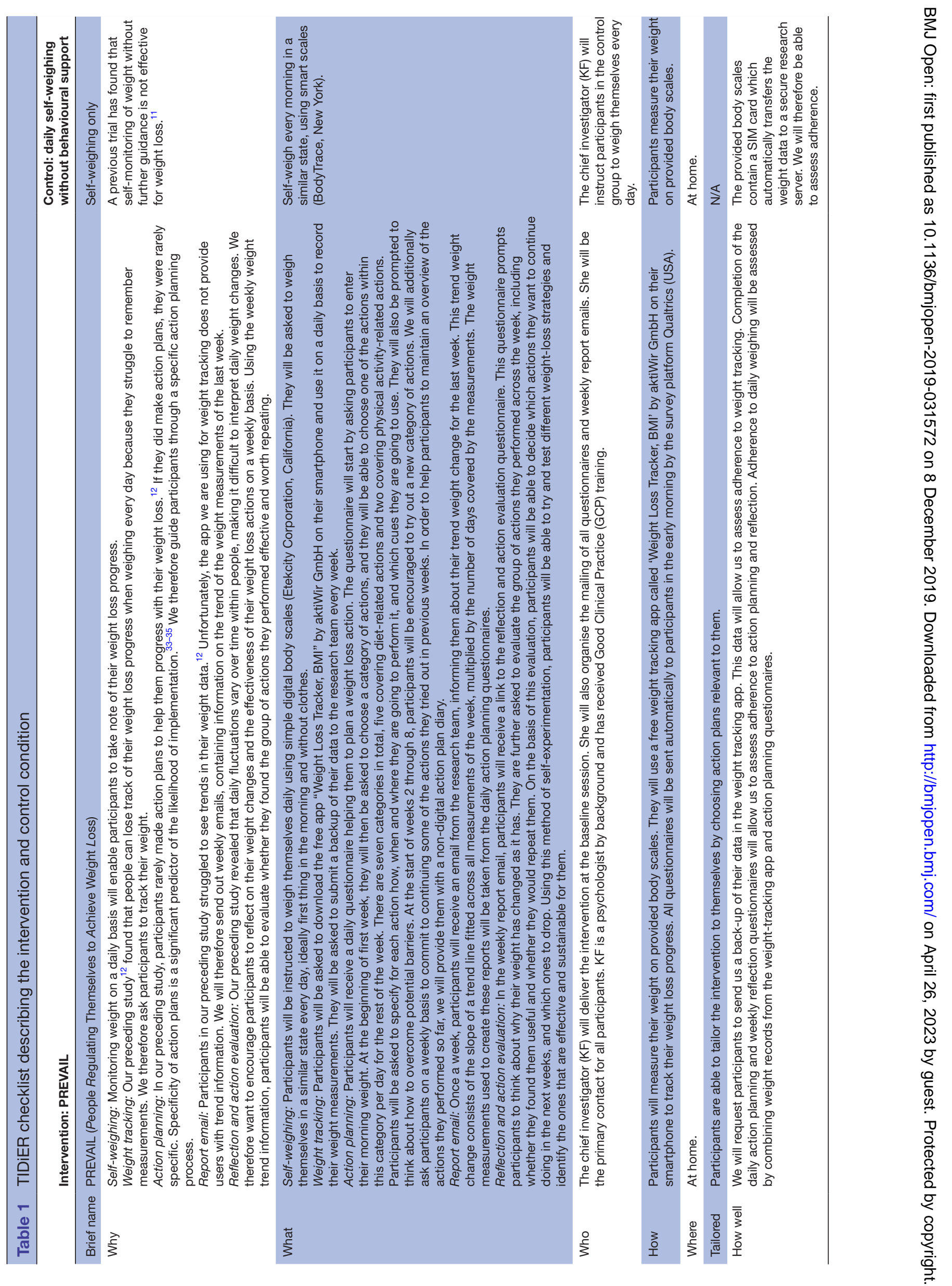
Table 2 Schedule of measurements

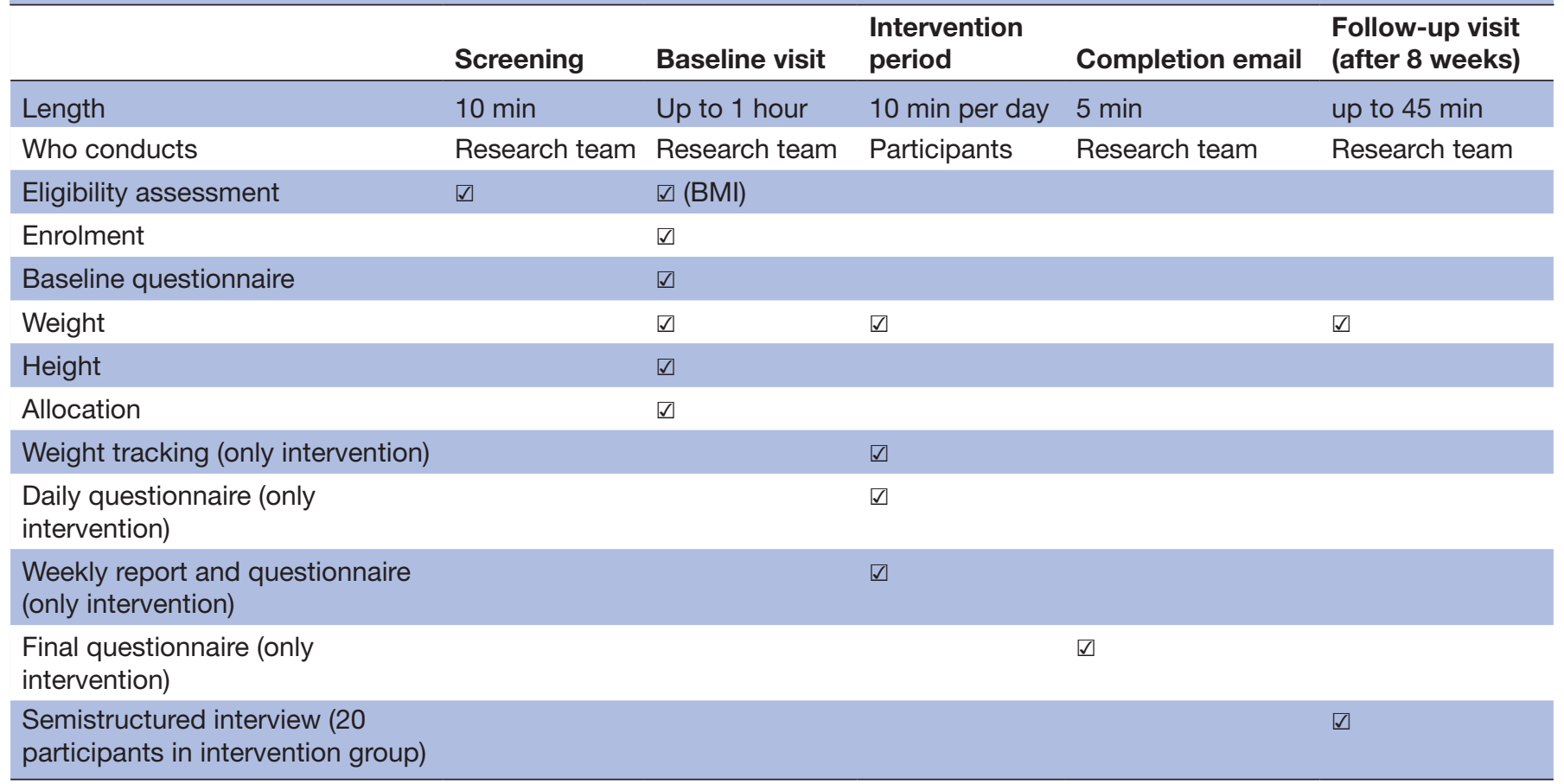

BMI, body mass index.

adherence score will be calculated averaging adherence rates across all intervention components.

\section{Evaluation of intervention components}

Using the data from the final online questionnaire, we will calculate means and SDs of the ratings for each intervention component.

\section{Retention}

The daily questionnaire and weekly report emails will act as prompts for the participants to engage with the intervention. At the end of the follow-up visit, participants will receive a $£ 35$ one 4 all gift card.

There are no criteria for withdrawal other than participants' request to withdraw. Participants can also ask to withdraw their collected data. We will ask participants wishing to withdraw whether they are willing to attend the final follow-up and take part in an exit interview to understand the reasons for their discontinuation.

\section{Statistical analyses}

The statistical analysis of the primary outcome, effectiveness of the intervention for weight loss, will be carried out on the basis of intention-to-treat (ITT). That is, after randomisation, participants will be analysed according to their allocated intervention group. We will endeavour to obtain full follow-up data on every participant to allow full ITT analysis. A linear regression, predicting weight at 8 weeks' follow-up while adjusting for baseline weight and GP practice, will assess the effect of condition. ${ }^{37}$ We will assess the sensitivity of the analysis to different assumptions about missing data using a variety of imputation methods, including baseline observation carried forward analysis and an analysis of participants completing follow-up. A final analysis will impute the last home-measured weight for people who did not attend the final follow-up. All tests will be done at a $5 \%$ twosided significance level.

Secondary outcomes will be analysed in several ways. Adherence rates will be assessed and compared between the experimental and control condition. Moderator analyses will assess the effect of adherence, highest educational qualification, liking of weighing at baseline, and overall intervention rating at follow-up (only intervention condition) on weight change.

Further exploratory analyses may be added post-hoc based on preliminary findings. The statistical analysis plan can be found in online supplementary appendix 2 .

\section{Qualitative study}

All interview audio-recordings will be transcribed and entered into the NVivo software package (QSR International) for qualitative data analysis. Framework analysis according to Ritchie and Spencer ${ }^{38}$ will assess the participants' experiences and perceptions of the different intervention components. The findings will be put into context with the results from the final questionnaire. Inductive thematic analysis following Braun and Clarke ${ }^{39}$ will explore additional themes, including barriers and unmet needs. One researcher with training in qualitative methods will perform coding for all interviews. 


\section{Trial management group}

The day-to-day management and operation of the study will be coordinated by KF. A Trial Management Group (TMG), consisting of the authors of this paper, will have oversight of the trial. The TMG will be responsible for the monitoring of all aspects of the trial's conduct and progress and will ensure that the protocol is adhered to and that appropriate action is taken to safeguard participants and the quality of the trial itself. The TMG will meet regularly throughout the course of the trial.

\section{Adverse events}

This is a low risk trial where it is implausible that the intervention will lead to differences in the occurrence of adverse events so we decided that it was inappropriate to burden participants to collect and record these.

\section{Trial monitoring}

This is a short trial with no adverse event monitoring or stopping rules so we deemed that a trial steering committee and a data monitoring committee were unnecessary.

\section{Data management}

Data will be kept in accordance with GCP, the Data Protection Act 2018 and General Data Protection Regulation. Two separate databases will be created, one containing all participant identifiable information, the other capturing all outcome data in an anonymised manner, using a unique participant ID. Weight, height and body composition measurements will be entered into the second database by the researcher. Data from the online questionnaires will be downloaded from Qualtrics and added to the second database. The two databases as well as the anonymised recordings and transcriptions from follow-up interviews will be stored on the secure departmental drive and will only be accessible by members of the TMG. After a lay summary of results has been sent out to participants, the database with participant identifiable data will be destroyed. We will retain the anonymised database for future secondary analyses.

Direct access to study data will be granted to authorised representatives from the sponsor for monitoring and/or audit of the study to ensure compliance with regulations. This access, the reason for it and who has authorised it will be recorded by the TMG. Otherwise, confidentiality will be maintained and no-one outside the TMG will have access to the database.

\section{Ethics and dissemination}

Any substantial changes to the protocol will be submitted as an amendment to the NHS National Research Ethics Committee and the Health Research Authority, as well as the Sponsor. On completion of the trial, KF will submit an End of Study notification and final report tothese institutions.

We intend to publish the results of this study in peerreviewed journals, regardless of the nature of the outcome. Authorship will be determined in accordance with the
International Committee of Medical Journal Editors (ICMJE) guidelines. We will also present our findings at national and international conferences, and publicise our publications through the departments' online presence. Participants will be informed of the trial results through an information sheet prepared for a lay audience. We will also inform our patients and public involvement panel members about the findings of the study through their regular newsletter.

Twitter Jamie Hartmann-Boyce @jhb19

Acknowledgements We would like to acknowledge and thank the members of the public who have helped design the PREVAlL intervention. We thank members of the department who have contributed in expert brainstorming sessions to the development of the intervention. We would like to thank Carmen Piernas and Rhiannon Edwards for creating the randomisation sequence and the randomisation envelopes. We thank the Clinical Trials Unit of the Nuffield Department of Primary Care Health Sciences for their help with setting up this study and support in conducting the trial.

Contributors KF, JHB, SJ and PA contributed to the design of the intervention and this study. KF led the preparation of the trial. All authors commented and worked on this paper. The sponsor has reviewed all participant-facing documents as part of the ethics application.

Funding This research is funded by the National Institute for Health Research (NIHR) Collaboration for Leadership in Applied Health Research and Care Oxford (CLAHRC) at Oxford Health NHS Foundation Trust. KF's time on this project is funded by NIHR CLAHRC Oxford at Oxford Health NHS Foundation Trust, Wolfson College, University of Oxford (Oxford-Wolfson Marriott-Primary Care Graduate Scholarship), and NIHR School for Primary Care Research (NIHR SPCR). JHB's, SJ's and PA's time on this project is funded by the NIHR Oxford Biomedical Research Centre (BRC) and Oxford CLAHRC. PA and SJ are NIHR senior investigators.

Disclaimer The views expressed are those of the authors and not necessarily those of the NHS, the NIHR, the Wellcome Trust or the Department of Health and Social Care. The sponsor is not involved in the collection, management, analysis and interpretation of data; writing of the report; and the decision to submit the report for publication of the study.

Competing interests None declared.

Patient consent for publication Not required.

Ethics approval This trial was reviewed and approved by the NHS National Research Ethics Committee and the Health Research Authority (reference number: 18/SC/0482).

Provenance and peer review Not commissioned; externally peer reviewed.

Open access This is an open access article distributed in accordance with the Creative Commons Attribution 4.0 Unported (CC BY 4.0) license, which permits others to copy, redistribute, remix, transform and build upon this work for any purpose, provided the original work is properly cited, a link to the licence is given, and indication of whether changes were made. See: https://creativecommons.org/ licenses/by/4.0/.

ORCID iDs

Kerstin Frie http://orcid.org/0000-0002-4717-5874

Jamie Hartmann-Boyce https://orcid.org/0000-0001-9898-3049

Susan A Jebb https://orcid.org/0000-0001-9190-2920

Paul Aveyard http://orcid.org/0000-0002-1802-4217

\section{REFERENCES}

1 Gokee-Larose J, Gorin AA, Wing RR. Behavioral self-regulation for weight loss in young adults: a randomized controlled trial. Int J Behav Nutr Phys Act 2009;6.

2 Oshima Y, Matsuoka Y, Sakane N. Effect of weight loss program using self-weighing twice a day and feedback in overweight and obese subject: a randomized controlled trial. Obes Res Clin Pract 2013;7:e361-6.

3 Helander EE, Vuorinen A-L, Wansink B, et al. Are breaks in daily selfweighing associated with weight gain? PLoS One 2014;9:e113164. 
4 Steinberg DM, Tate DF, Bennett GG, et al. The efficacy of a daily self-weighing weight loss intervention using smart scales and e-mail. Obesity 2013;21:1789-97.

5 Burke LE, Wang J, Sevick MA. Self-Monitoring in weight loss: a systematic review of the literature. J Am Diet Assoc 2011;111:92-102.

6 Kanfer FH. Self-Monitoring: methodological limitations and clinical applications. J Consult Clin Psychol 1970;35:148-52.

7 Boutelle K. Letters to the editor. J Nutr Educ Behav 2006;38:131.

8 Kanfer FH, Karoly P. Self-control: a behavioristic excursion into the lion's den. Behav Ther 1972;3:398-416.

9 Hartmann-Boyce J, Boylan A-M, Jebb SA, et al. Experiences of self-monitoring in self-directed weight loss and weight loss maintenance: systematic review of qualitative studies. Qual Health Res 2019:29:124-34.

10 Daskalova N, Desingh K, Papoutsaki A, et al. Lessons learned from two cohorts of personal informatics Self-Experiments. Proceedings of the ACM on interactive, mobile, wearable and ubiquitous technologies 2017;1.

11 Madigan CD, Jolly K, Lewis AL, et al. A randomised controlled trial of the effectiveness of self-weighing as a weight loss intervention. Int $J$ Behav Nutr Phys Act 2014;11.

12 Frie K, Hartmann-Boyce J, Jebb S, et al, eds. The role of selfregulation in and barriers to self-monitoring of weight. Galway, Ireland: EHPS, 2018.

13 Pacanowski CR, Levitsky DA. Frequent Self-Weighing and visual feedback for weight loss in overweight adults. J Obes 2015;2015:763680:9.

14 Leahey TM, Thomas G, Fava JL, et al. Adding evidence-based behavioral weight loss strategies to a statewide wellness campaign: a randomized clinical trial. Am J Public Health 2014;104:1300-6.

15 Bennett GG, Steinberg D, Askew S, et al. Effectiveness of an APP and provider counseling for obesity treatment in primary care. Am J Prev Med 2018:55:777-86.

16 Dombrowski SU, Endevelt R, Steinberg DM, et al. Do more specific plans help you lose weight? examining the relationship between plan specificity, weight loss goals, and plan content in the context of a weight management programme. Br J Health Psychol 2016;21:989-1005

17 Benyamini Y, Geron R, Steinberg DM, et al. A structured intentions and action-planning intervention improves weight loss outcomes in a group weight loss program. Am J Health Promot 2013;28:119-27.

18 Lin M, Mahmooth Z, Dedhia N, et al. Tailored, interactive text messages for enhancing weight loss among African American adults: the TRIMM randomized controlled trial. Am J Med 2015;128:896-904.

19 Huisman S, de Gucht V, Maes S, et al. Self-regulation and weight reduction in patients with type 2 diabetes: a pilot intervention study. Patient Educ Couns 2009;75:84-90.

20 Mann DM, Palmisano J, Lin JJ. A pilot randomized trial of technology-assisted goal setting to improve physical activity among primary care patients with prediabetes. Prev Med Rep 2016;4:107-12.

21 Steinberg DM, Levine EL, Askew S, et al. Daily text messaging for weight control among racial and ethnic minority women: randomized controlled pilot study. J Med Internet Res 2013;15:e244.

22 Lin P-H, Wang Y, Levine E, et al. A text messaging-assisted randomized lifestyle weight loss clinical trial among overweight adults in Beijing. Obesity 2014;22:E29-37.
23 Huisman S, Maes S, De Gucht VJ, et al. Low goal ownership predicts drop-out from a weight intervention study in overweight patients with type 2 diabetes. Int J Behav Med 2010;17:176-81.

24 Deci EL, Ryan RM. The "what" and "why" of goal pursuits: human needs and the self-determination of behavior. Psychol Inq 2000;11:227-68.

25 Luszczynska A, Sobczyk A, Abraham C. Planning to lose weight: randomized controlled trial of an implementation intention prompt to enhance weight reduction among overweight and obese women. Health Psychol 2007;26:507-12.

26 Linde JA, Jeffery RW, Crow SJ, et al. The tracking study: description of a randomized controlled trial of variations on weight tracking frequency in a behavioral weight loss program. Contemp Clin Trials 2015;40:199-211.

27 Morrison LG, Hargood C, Lin SX, et al. Understanding usage of a hybrid website and smartphone APP for weight management: a mixed-methods study. J Med Internet Res 2014;16:e201.

28 Lally P, Chipperfield A, Wardle J. Healthy habits: efficacy of simple advice on weight control based on a habit-formation model. Int $J$ Obes 2008;32:700-7.

29 Madigan CD, Daley AJ, Lewis AL, et al. Is self-weighing an effective tool for weight loss: a systematic literature review and meta-analysis. Int J Behav Nutr Phys Act 2015;12.

30 Burke LE, Conroy MB, Sereika SM, et al. The effect of electronic self-monitoring on weight loss and dietary intake: a randomized behavioral weight loss trial. Obesity 2011;19:338-44.

31 Carter MC, Burley VJ, Nykjaer C, et al. Adherence to a smartphone application for weight loss compared to website and paper diary: pilot randomized controlled trial. J Med Internet Res 2013;15:e32.

32 Frie K, Hartmann-Boyce J, Jebb S, et al. Insights from Google play store user reviews for the development of weight loss Apps: MixedMethod analysis. JMIR Mhealth Uhealth 2017;5:e203.

33 de Vet E, Oenema A, Brug J. More or better: do the number and specificity of implementation intentions matter in increasing physical activity? Psychol Sport Exerc 2011;12:471-7.

34 Gollwitzer PM, Sheeran P. Implementation intentions and goal achievement: a Meta-analysis of effects and processes. advances in experimental social psychology volume 38. Adv Exp Soc Psychol 2006;38:9-119.

35 Hagger MS, Luszczynska A. Implementation intention and action planning interventions in health contexts: state of the research and proposals for the way forward. Appl Psychol Health Well Being 2014;6:1-47.

36 Hoffmann TC, Glasziou PP, Boutron I, et al. Better reporting of interventions: template for intervention description and replication (TIDieR) checklist and guide. BMJ 2014;348:g1687.

37 Vickers AJ, Altman DG. Statistics notes: analysing controlled trials with baseline and follow up measurements. BMJ 2001;323:1123-4.

38 Ritchie J, Spencer L. Qualitative data analysis for applied policy research. In: Huberman A, Miles M, eds. The qualitative researcher's companion. Thousand Oaks: SAGE Publications, Inc, 2002.: 305-29.

39 Braun V, Clarke V. Thematic analysis. In: Cooper H, Camic PM, Long $\mathrm{DL}$, eds. APA Handbook of research methods in psychology, vol 2 research designs: quantitative, qualitative, neuropsychological, and biological. Washington, DC, US: American Psychological Association, 2012: 57-71. 